\title{
Higher Mellin Moments for Charged Current DIS
}

\section{Rogal* and S. Moch}

Deutsches Elektronensynchrotron DESY

Platanenallee 6, D-15738 Zeuthen, Germany

E-mail: Mikhail.Rogal@desy.de, Sven-Olaf.Moch@desy.de

\begin{abstract}
We report on our recent results for deep-inelastic neutrino $(v)$-proton $(P)$ scattering. We have computed the perturbative QCD corrections to three loops for the charged current structure functions $F_{2}, F_{L}$ and $F_{3}$ for the combination $v P-\bar{v} P$. In leading twist approximation we have calculated the first six odd-integer Mellin moments in the case of $F_{2}$ and $F_{L}$ and the first six even-integer moments in the case of $F_{3}$. As a new result we have obtained the coefficient functions to $\mathscr{O}\left(\alpha_{s}^{3}\right)$ and we have found the corresponding anomalous dimensions to agree with known results in the literature.
\end{abstract}

XI International Workshop on Advanced Computing and Analysis Techniques in Physics Research April 23-27 2007

Amsterdam, the Netherlands

${ }^{*}$ Speaker. 


\section{Introduction}

In our recent research, we extended the program of calculating higher order perturbative QCD corrections to the structure functions of charged current deep-inelastic scattering (DIS). Our studies are motivated by the increasingly accurate measurements of neutral and charged current cross sections at HERA with a polarized beam of electrons and positrons [1, 2, 3]. At the same time we are also able to quantitatively improve predictions for physics at the front-end of a neutrino-factory, see e.g. Ref. [4]. To be specific, we consider neutrino $(v)$-proton $(P)$ scattering in the combination $v P-\bar{v} P$, which corresponds to charged lepton-proton DIS as far as QCD corrections are concerned. Following Refs. [5, 6, 7, 8, 9] we compute the perturbative QCD (pQCD) predictions to three-loop accuracy for a number of fixed Mellin moments of the structure functions $F_{2}, F_{L}$ and $F_{3}$.

Within the framework of the operator product expansion (OPE), and working in Mellin space, $F_{2}^{v P-\bar{v} P}$ and $F_{L}^{v P-\bar{v} P}$ are functions of odd Mellin moments only, while only even moments contribute to $F_{3}^{v P-\bar{v} P}$. This is opposite to the case of the neutral current structure functions where only even Mellin moments contribute, and to the charged current case for $v P+\bar{v} P$ scattering [10], which is defined through the OPE for odd Mellin moments only. In the latter results for $F_{2}^{v P+\bar{v} P}$ and $F_{L}^{\nu P+\bar{v} P}$ to three-loops can also be directly checked in electromagnetic DIS [11, 12] while parameterizations for $F_{3}^{\nu P+\bar{v} P}$ to three-loop accuracy are given in Ref. [13].

\section{General formalism}

We consider unpolarized inclusive deep-inelastic lepton-nucleon scattering,

$$
l(k)+\operatorname{nucl}(p) \rightarrow l^{\prime}\left(k^{\prime}\right)+X
$$

where $l(k), l^{\prime}\left(k^{\prime}\right)$ are leptons of momenta $k$ and $k^{\prime}, \operatorname{nucl}(p)$ denotes a nucleon of momentum $p$ and $X$ stands for all hadronic states allowed by quantum number conservation. In our research we are concentrating on charged current neutrino $(v)$-proton $(P)$ scattering, i.e. $v P, \bar{v} P$ via $W^{ \pm}$boson exchange. As is well known, the differential cross section for the reaction (2.1) can be written as a product of leptonic $L_{\mu \nu}$ and hadronic $W_{\mu \nu}$ tensors

$$
d \sigma \propto L^{\mu v} W_{\mu v}
$$

with $L^{\mu v}$ for electroweak or pure electromagnetic gauge boson exchange given in the literature, see e.g. Ref. [14]. The hadronic tensor $W^{\mu v}$ in Eq. (2.2) can be written in terms of so called structure functions $F_{i}, i=2,3, L$.

We are interested in the Mellin moments of structure functions, defined as

$$
F_{i}\left(n, Q^{2}\right)=\int_{0}^{1} d x x^{n-2} F_{i}\left(x, Q^{2}\right), \quad i=2, L
$$

and for $F_{3}\left(n, Q^{2}\right)$ one has similar relation with $n$ replaced by $n+1$ on the r.h.s. of Eq. (2.3). Here $Q^{2}=-q^{2}>0, q=k-k^{\prime}$ and $x$ is the Bjorken scaling variable defined as $x=Q^{2} /(2 p \cdot q)$ with $0<x \leq 1$. 
With the help of the optical theorem and Cauchy's theorem from complex analysis one can relate the Mellin moments of structure functions to the parameters of the OPE for the nucleon forward Compton amplitude $T_{\mu \nu}$ :

$$
F_{i}\left(n, Q^{2}\right)=C_{i, \mathrm{~ns}}\left(n, \frac{Q^{2}}{\mu^{2}}, \alpha_{s}\right) A_{\text {nucl }}^{\mathrm{ns}}\left(n, \mu^{2}\right), \quad i=2,3, L
$$

and the OPE for $T_{\mu \nu}$ reads as

$$
\begin{aligned}
T_{\mu \nu}= & 2 \sum_{n} \omega^{n}\left[e_{\mu \nu} C_{L, \mathrm{~ns}}\left(n, \frac{Q^{2}}{\mu^{2}}, \alpha_{s}\right)+d_{\mu \nu} C_{2, \mathrm{~ns}}\left(n, \frac{Q^{2}}{\mu^{2}}, \alpha_{s}\right)\right. \\
& \left.+\mathrm{i} \varepsilon_{\mu \nu \alpha \beta} \frac{p^{\alpha} q^{\beta}}{p \cdot q} C_{3, \mathrm{~ns}}\left(n, \frac{Q^{2}}{\mu^{2}}, \alpha_{s}\right)\right] A_{\mathrm{nucl}}^{\mathrm{ns}}\left(n, \mu^{2}\right)+\text { higher twists }
\end{aligned}
$$

where higher twist contributions are omitted. $C_{i, \text { ns }}$ denote the Wilson coefficients which are calculable in pQCD and $A_{\text {nucl }}^{\text {ns }}$ are matrix elements of quark non-singlet operators. The latter are not calculable in $\mathrm{pQCD}$, rather they have to be extracted from experimental data. We restrict ourselves to quark non-singlet (ns) operators only since only these give nonvanishing contributions in the combination $v P-v N$ (see Ref. [10] for details).

Eq. (2.4) provides the basis to obtain Mellin moments of DIS structure functions in our approach by means of the OPE and the optical theorem. Furthermore, from the careful examination of the symmetry properties of the forward Compton amplitude $T_{\mu \nu}$ and, related, the underlying Feynman diagrams, one can convince oneself that for the charged current $v P-v N$ DIS, one encounters functions of only odd $n$ for $F_{2}$ and $F_{L}$ and, functions of only even $n$ for $F_{3}$, respectively [10].

The pQCD calculation of Wilson coefficients $C_{i, \text { ns }}$ proceeds through the following steps. From the first principles we calculate the partonic forward Compton amplitude $t_{\mu \nu}$. The partonic equivalent of the OPE Eq. (2.5) for $t_{\mu \nu}$ contains the same coefficients $C_{i, \text { ns }}$ as in Eq. (2.5) and quark matrix elements $A_{\mathrm{q}}^{\mathrm{ns}}$. Projection on the $n^{\prime}$ th Mellin moment of OPE and on the $i$ 'th parton invariant $(i=2,3, L)$ with the help of the operator $\mathscr{P}_{n, i}^{\mu v}$ we get

$$
t_{i, \mathrm{~ns}}\left(n, \frac{Q^{2}}{\mu^{2}}, \alpha_{s}, \varepsilon\right) \equiv \mathscr{P}_{n, i}^{\mu v} t_{\mu v}=C_{i, \mathrm{~ns}}\left(n, \frac{Q^{2}}{\mu^{2}}, \alpha_{s}, \varepsilon\right) Z_{\mathrm{ns}}\left(\alpha_{s}, \frac{1}{\varepsilon}\right) A_{\mathrm{q}}^{\mathrm{ns}, \text { tree }}(n, \varepsilon) .
$$

Both sides of Eq. (2.6) are renormalized. In particular the renormalization of the local quark operator matrix element $A_{\mathrm{q}}^{\text {ns }}$ gives rise to the factor $Z_{\mathrm{ns}}$ on the r.h.s. of Eq. (2.6). This equation is our starting point for an iterative determination of the coefficient functions $C_{i, \mathrm{~ns}}$ and the anomalous dimension $\gamma_{\mathrm{ns}}$. The latter appears in a series expansion of $Z_{\mathrm{ns}}$ in powers of the strong coupling $\alpha_{s}$ and negative powers of the parameter $\varepsilon$ of dimensional regularization, $D=4-2 \varepsilon$. The $C_{i \text {,ens }}$ on the other hand are expanded in $\alpha_{s}$ and in positive powers of $\varepsilon$. Thus the 1.h.s. of Eq. (2.6) leads to a well defined determination of $C_{i, \text { ens }}$ and $Z_{\text {ens }}$ in pQCD.

\section{Calculation and checks}

In the previous section, we have briefly explained the method to obtain Mellin moments of the DIS charged current structure functions $F_{2}^{v P-\bar{v} P}, F_{3}^{v P-\bar{v} P}$ and $F_{L}^{v P-\bar{v} P}$ together with their respective 
coefficient functions and anomalous dimensions. To that end we have calculated the Lorentz invariants of the parton Compton amplitude $t_{i, \text { ens }}, i=2,3, L$, as given in the 1.h.s. of Eq. (2.6). Due to the large number of diagrams involved in the calculations up to order $\alpha_{s}^{3}$ sufficient automatization is necessary. First of all, we have generated 3633 diagrams up to three loops with the program QGRAF [15]. For all further calculations we have relied on the latest version of the symbolic manipulation program FORM $[16,17]$.

For the treatment of QGRAF output, such as analysis of the topologies, the explicit implementation of Feynman rules etc. we have adapted a dedicated FORM procedure conv.prc from previous work, e.g. Ref. [12]. Most importantly, this procedure tries to exploit as many symmetry properties of the original Feynman diagrams as possible in order to reduce their total number.

For the calculation of the color factors for each Feynman diagram we have used the FORM package color.h [18]. The actual calculation of the Mellin moments of the Feynman integrals has made use of the FORM version of MinCER [19]. Finally, on top of Mincer and Minos [6] some shell scripts managed the automatic runs of both programs for different parts of the calculation.

We have performed various checks on our computation. Most prominently, we have kept all powers of the gauge parameter $\xi$ throughout the entire calculation for Mellin moments $n \leq 10$ to check that any $\xi$-dependence vanishes in our final results. The Mellin moments with $n>10$ were calculated without gauge parameter to facilitate the computations which become increasingly more complicated for higher Mellin $n$ values. For these moments we also used TFORM [20], the multi-threaded version of FORM. On machines with multi-core processors this leads to a significant speed up of our calculations, e.g. a speed-up of $\simeq 5$ on a two-core four processor machine.

We agree with the literature as far as the two-loop coefficient functions [21, 22, 23, 24, 25] and the three-loop anomalous dimensions [26] are concerned. In addition, for the first Mellin moment of the coefficient function $C_{2, \text { ens }}$ we have obtained exactly $C_{2, \text { ens }}=1$ to all orders in $\alpha_{s}$ which is in agreement with the Adler sum rule for DIS structure functions,

$$
\int_{0}^{1} \frac{d x}{x}\left(F_{2}^{v P}\left(x, Q^{2}\right)-F_{2}^{v N}\left(x, Q^{2}\right)\right)=2 .
$$

The Adler sum rule measures the isospin of the nucleon in the quark-parton model and does not receive any perturbative or non-perturbative corrections in QCD, see e.g. Ref. [27]. Therefore, this result is another important check of the correctness of our results.

\section{Conclusions}

We have reported on new results for Mellin moments of the charged current DIS structure functions $F_{2}^{v P-\bar{v} P}, F_{L}^{v P-\bar{v} P}$ and $F_{3}^{v P-\bar{v} P}$ including the perturbative QCD corrections to three loops. In the former case $\left(F_{2}, F_{L}\right)$ we have computed the first six odd-integer Mellin moments while in the latter case $\left(F_{3}\right)$, the first six even-integer moments have been obtained. The results for $F_{2, L}^{v P-\bar{v} P} n=1,3,5,7,9$ and for $F_{3}^{v P-\bar{v} P} n=2,4,6,8,10$ are available in Ref. [10]. Results for $n=11$ in the former case and for $n=12$ in the latter will be published elsewhere. Finally, the discussion of phenomenological consequences of our Mellin space results along with approximate parameterizations the coefficient functions in $x$ are deferred to Ref. [28]. 


\section{References}

[1] ZEUS Collaboration, S. Chekanov et al., Measurement of high- $Q^{2}$ charged current cross sections in $e^{+}$p deep inelastic scattering at HERA, Eur. Phys. J. C32 (2003) 1-16, [hep-ex/ 0307043 ].

[2] H1 Collaboration, C. Adloff et al., Measurement and QCD analysis of neutral and charged current cross sections at HERA, Eur. Phys. J. C30 (2003) 1-32, [hep-ex/ 0304003 ].

[3] H1 Collaboration, A. Aktas et al., First measurement of charged current cross sections at HERA with longitudinally polarized positrons, Phys. Lett. B634 (2006) 173-179, [hep-ex/0 512060$].$

[4] M. L. Mangano et al., Physics at the front-end of a neutrino factory: A quantitative appraisal, hep-ph/0105155.

[5] S. A. Larin, T. van Ritbergen, and J. A. M. Vermaseren, The next next-to-leading QCD approximation for nonsinglet moments of deep inelastic structure functions, Nucl. Phys. B427 (1994) 41-52.

[6] S. A. Larin, P. Nogueira, T. van Ritbergen, and J. A. M. Vermaseren, The three-loop QCD calculation of the moments of deep inelastic structure functions, Nucl. Phys. B492 (1997) 338-378, [hep-ph/9605317].

[7] A. Retey and J. A. M. Vermaseren, Some higher moments of deep inelastic structure functions at next-to-next-to-leading order of perturbative QCD, Nucl. Phys. B604 (2001) 281-311, [hep-ph/0007294].

[8] S. Moch, J. A. M. Vermaseren, and A. Vogt, Next-to-next-to-leading order QCD corrections to the photon's parton structure, Nucl. Phys. B621 (2002) 413-458, [hep-ph/0110331].

[9] J. Blümlein and J. A. M. Vermaseren, The 16th moment of the non-singlet structure functions $F_{2}\left(x, q^{2}\right)$ and $F_{L}\left(x, q^{2}\right)$ to $O\left(\alpha_{s}^{3}\right)$, Phys. Lett. B606 (2005) 130-138, [hep-ph / 0411111 ].

[10] S. Moch and M. Rogal, Charged current deep-inelastic scattering at three loops, arXiv:0704.1740 [hep-ph].

[11] S. Moch, J. A. M. Vermaseren, and A. Vogt, The longitudinal structure function at the third order, Phys. Lett. B606 (2005) 123-129, [hep-ph/ 0411112$].$

[12] J. A. M. Vermaseren, A. Vogt, and S. Moch, The third-order QCD corrections to deep-inelastic scattering by photon exchange, Nucl. Phys. B724 (2005) 3-182, [hep-ph/ 0504242$].$

[13] A. Vogt, S. Moch, and J. Vermaseren, Third-order QCD results on form factors and coefficient functions, Nucl. Phys. Proc. Suppl. 160 (2006) 44-50, [hep-ph / 0608307$].$

[14] Particle Data Group Collaboration, W. M. Yao et al., Review of particle physics, J. Phys. G33 (2006) 1-1232.

[15] P. Nogueira, Automatic Feynman graph generation, J. Comput. Phys. 105 (1993) 279-289.

[16] J. A. M. Vermaseren, Tuning FORM with large calculations, Nucl. Phys. Proc. Suppl. 116 (2003) 343-347, [hep-ph/0211297].

[17] J. A. M. Vermaseren and M. Tentyukov, What is new in FORM, Nucl. Phys. Proc. Suppl. 160 (2006) 38-43.

[18] T. van Ritbergen, A. N. Schellekens, and J. A. M. Vermaseren, Group theory factors for Feynman diagrams, Int. J. Mod. Phys. A14 (1999) 41-96, [hep-ph/9802376].

[19] S. A. Larin, F. V. Tkachov, and J. A. M. Vermaseren, The FORM version of MINCER, NIKHEF-H-91-18. 
[20] M. Tentyukov and J. A. M. Vermaseren, The multithreaded version of FORM, hep-ph/0 702279.

[21] W. L. van Neerven and E. B. Zijlstra, Order $\alpha_{s}^{2}$ contributions to the deep inelastic Wilson coefficient, Phys. Lett. B272 (1991) 127-133.

[22] E. B. Zijlstra and W. L. van Neerven, Contribution of the second order gluonic Wilson coefficient to the deep inelastic structure function, Phys. Lett. B273 (1991) 476-482.

[23] E. B. Zijlstra and W. L. van Neerven, Order $\alpha_{s}^{2}$ correction to the structure function $F_{3}\left(x, q^{2}\right)$ in deep inelastic neutrino - hadron scattering, Phys. Lett. B297 (1992) 377-384.

[24] E. B. Zijlstra and W. L. van Neerven, Order $\alpha_{s}^{2} Q C D$ corrections to the deep inelastic proton structure functions $F_{2}$ and $F_{L}$, Nucl. Phys. $\mathbf{B 3 8 3}$ (1992) 525-574.

[25] S. Moch and J. A. M. Vermaseren, Deep inelastic structure functions at two loops, Nucl. Phys. B573 (2000) 853-907, [hep-ph/9912355].

[26] S. Moch, J. A. M. Vermaseren, and A. Vogt, The three-loop splitting functions in QCD: The non-singlet case, Nucl. Phys. $\mathbf{6 6 8 8}$ (2004) 101-134, [hep-ph / 0403192$].$

[27] Y. L. Dokshitzer, G. Marchesini, and B. R. Webber, Dispersive approach to power-behaved contributions in QCD hard processes, Nucl. Phys. B469 (1996) 93-142, [hep-ph/9512336].

[28] S. Moch, M. Rogal, and A. Vogt, Differences between charged current coefficient functions, DESY 07-048. 